\title{
A Comprehensive Review of Lyme Disease, An Approach to Lyme in Iran
}

\author{
SShahbeigi ${ }^{1 *}$, ANajjari ${ }^{2}$, NFayyazi ${ }^{3}$, SANojoumi ${ }^{4}$, SRNaddaf ${ }^{5}$ and HPakdaman ${ }^{6}$ \\ ${ }^{1}$ Brain Mapping Research Centre, Shahid Beheshti University, Neuroscience Research Centre, Iran University of Medical Sciences, Iran \\ ${ }^{2}$ Ministry of Health and Medical Education, Tehran, Iran \\ ${ }^{3}$ Neurology Research Group, Stem Cell Association, Tehran, Iran \\ ${ }^{4}$ Seyed Ali Nojoumi, Microbiology Research Center, Pasteur Institute of Iran, Tehran, Iran \\ ${ }^{5}$ Department of Parasitology, Pasteur Institute of Iran, Tehran, Iran \\ ${ }^{6}$ Professor of Neurology, Shahid Beheshti Medical University, Iran
}

*Corresponding author: SShahbeigi, Brain Mapping Research Centre, Shahid Beheshti University, Neuroscience Research Centre,

Iran University of Medical Sciences, Iran

ARTICLE INFO

Received: 幽 July 16, 2020

Published: 幽 August 07, 2020

Citation: SShahbeigi, ANajjari, NFayyazi, SANojoumi, SRNaddaf, HPakdaman. A Comprehensive Review of Lyme Disease, An Approach to Lyme in Iran. Biomed J Sci \& Tech Res 29(3)-2020. BJSTR. MS.ID.004796.

\section{ABSTRACT}

Lyme disease is the most prevalent vector-borne disease in the northern hemisphere. The causative spirochetes, different species of the Borrelia bourgorferi senso lato complex, are transmitted via the infective bites of the members of the genus Ixodes. Two different studies suggest that around 300,000 cases of Lyme disease occur annually in the United States, mostly from the Northeast and upper Midwest. Despite relatively high incidence of Lyme disease in northern countries, in Iran the disease occurrence is so rare. According to PubMed and Google, through careful searching, only three Lyme disease cases have been reported in Iran. Here, we intend to discuss the neurological manifestations of Lyme (Lyme Neuroborreliosis), followed by explaining about the diagnosis and finally discussing the treatment options of Lyme too.

Keywords: Borrelia Burgdorferi; Tick-borne;Spirochete; Lyme; Lyme Neuroborreliosis (LNB); Iran;Bannwarth Syndrome

\section{Introduction}

Lyme disease was first presented by Afzelius, a Swedish dermatologist in 1920. He described an atypical skin lesion termed erythema chronicum migrans. Then he realized that the skin lesion was caused by bites of hard-shelled Ixodes ticks. Two years later, two French clinicians presented a 58-year-old man who developed an enlarging erythroderma, 3 weeks after a tick bite on the left buttock accompanied by severe radicular pain. This disorder was named as Garin-Bujadoux-Bannwarth syndrome. The syndrome had been treated with penicillin by the 1950s[1]. In the USA, 32 children were diagnosed with relapsing large joint arthritis. They manifested a history of erythema chronicum migrans as well and then developed arthritis and some certain symptoms such as cardiac and neurologic involvements too[2]. In the early 1980s, it was indicated that this was due to infection with a family of tickborne spirochetes, named Borrelia burgdorferi[3]. It soon became clear that patients who developed EM also developed lymphocytic meningitis, cranial neuritis, and painful radiculoneuritis, resembling the patients infected in Europe. The disorders were thoroughly caused by B. burgdorferi sensustricto in the USA, but also the pathogen was exhibited in a minority of European cases. In Europe, B. gariniiis the most common pathogen in patients with neurologic disorders; however, $B$. afzeliiis very likely to manifest cutaneous involvement [4]. 


\section{Epidemiology}

Lyme disease is the most prevalent vector-borne disease in the northern hemisphere. The causative spirochetes, different species of the Borrelia bourgorferi senso lato complex, are transmitted via the infective bites of the members of the genus Ixodes. Two different studies suggest that around 300,000 cases of Lyme disease occur annually in the United States, mostly from the Northeast and upper Midwest [5]. Lyme disease peaks during summer, mainly in May and June. With increased awareness and improvement of diagnostic methods, the number of detected cases has risen since 1991.

\section{Lyme Disease in Iran}

According to PubMed and Google, upon careful searching, only three Lyme disease cases have been reported in Iran. The first one was a patient with a typical skin manifestation of Lyme in 1997. The second case in the 2004 from the Northern part of Iran also presented with EM presentation approved by the serological tests. The last case, in 2006, was a nine-year-old child with arthritis previously diagnosed by the dermatologic manifestation of Lyme [7-9]. Recently, the Lyme disease agents were detected by molecular methods in Ixodes ticks from the Caspian Sea littoral (Naddaf, personal communication).

\section{Clinical Presentation}

Lyme disease generally has 3 different stages:

a) Stage 1: after 3 to 32 days incubation period, a typical skin rash known as Erythema Migrans (EM) appears. The EM is accompanied by flu like symptoms such as fever, headache, and myalgia. The EM is a hallmark of Lyme disease manifested as an erythema more than $5 \mathrm{~cm}$ in diameter (Figure 1). The appearance of EM in endemic area is, indeed, sufficient for Lyme diagnosis. It is said that the EM is $100 \%$ specific and more sensitive (58 to $86 \%$ ) than serology tests in diagnosing Lyme disease. [10] Due to movement of spirochetes through lymphatics of the skin, the EM spreads centrifugally and causes an appearance of a target zone[11]. There are, sometimes multiple skin lesions, indicating that spirochetes might be disseminated in different skin area [12]. Migrating transitory pain in some joints, tendons, bones, and muscles is a common finding of early Lyme disease as well [13].

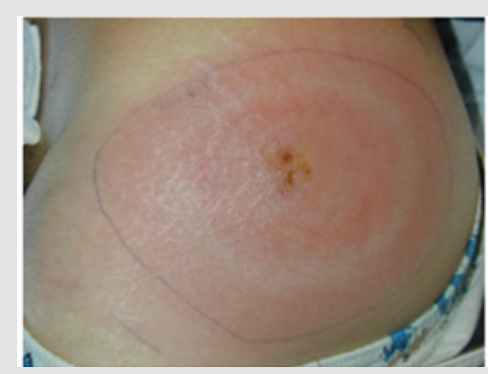

Figure 1: Erythema migrans in a patient with Lyme disease[14]. b) Stage 2: The neurologic and cardiac manifestation will be presented one to four months after the infection. Correspondingly, the neurologic and cardiac symptoms may be developed in around 15 and 8\%, respectively.

c) Stage 3: chronic neurological symptoms such as cranial neuritis and arthritic involvements may appear more than a few years later[14-18].

The CNS involvements can be classified into subgroups described below:

1. The CSF involvement, such as meningitis.

2. The brain or spinal cord parenchyma involvement such as encephalitis or encephalomyelitis.

3. The brain functions without involvement of brain structure, such as encephalopathy.

Peripheral Nervous System (PNS) disorders can be seen as well. It mayinvolve the axon, myelin, and both of them, too[1]. Generally, it has been thought that the involvement of brain parenchyma and spinal cord is rare. Below, we describe the different neurological manifestation of Lyme (LNB) more carefully.

\section{Cranial Neuritis}

A total of $5 \%$ to $10 \%$ cases involve cranial neuritis including 7 th cranial palsy and less frequently other types of cranial neuritis such as V, VIII, and the extraocular muscles nerves. Since the optic nerve is considered as a part of the CNS, it probably is more appropriately classified as a form of parenchymal CNS disease (encephalitis). The most important paraclinical findings on meningitis is the enhancement of meninges, cranial and spinal nerves. The most common cranial nerve involvement in LNB is facial palsy taking place in about $80 \%$ of cranial nerve involvement in LNB. In $1 / 3$ of patients with LNB, the facial nerve could be involved. The facial palsy is bilateral in $25 \%$ of cases [15]. The brain MRI shows diffuse enhancement of cranial nerves. In facts, the nodular enhancement of facial nerves is not usually seen. It is important to say that normal enhancement can be seen in long parts of the facial nerves [19-25], but in the clinical setting, attention should be paid to asymmetry or marked intensity of enhancement in different segments of facial nerves [26] (Figures 2\&3).

In a case presentation, the authors presented a 10-year-old patient with multiple cranial nerves involvements including the severe bilateral facial palsy andIII, V, VI, VIII, X and XII.MRI of the brain was demonstrated bilateral symmetric enhancement of multiple cranial nerves including the oculomotor (III), trigeminal (V), abducens (VI), facial (VII), vestibulo-cochlear (VIII), vagus (X) and hypoglossal (XII) as well as cervical cranial nerve roots (Figure 4). Cerebrospinal fluid testing showed pleocytosis133 cells/ $\mu \mathrm{L}$ (81\% lymphocytes, $13 \%$ neutrophils). CSF protein was increased at $177 \mathrm{mg} / \mathrm{dL}$ (normal $<60 \mathrm{mg} / \mathrm{dL}$ ) and normal glucose level. (43.2 mg/dL; (normal $45-79.3 \mathrm{mg} / \mathrm{dL}$ ) with a serum glucose 
of $91.9 \mathrm{mg} / \mathrm{dL}$. Bacterial culture plus testing for herpes simplex virus and enterovirus were negative. The patient was given 4-week IV ceftriaxone, and then he improved completely.
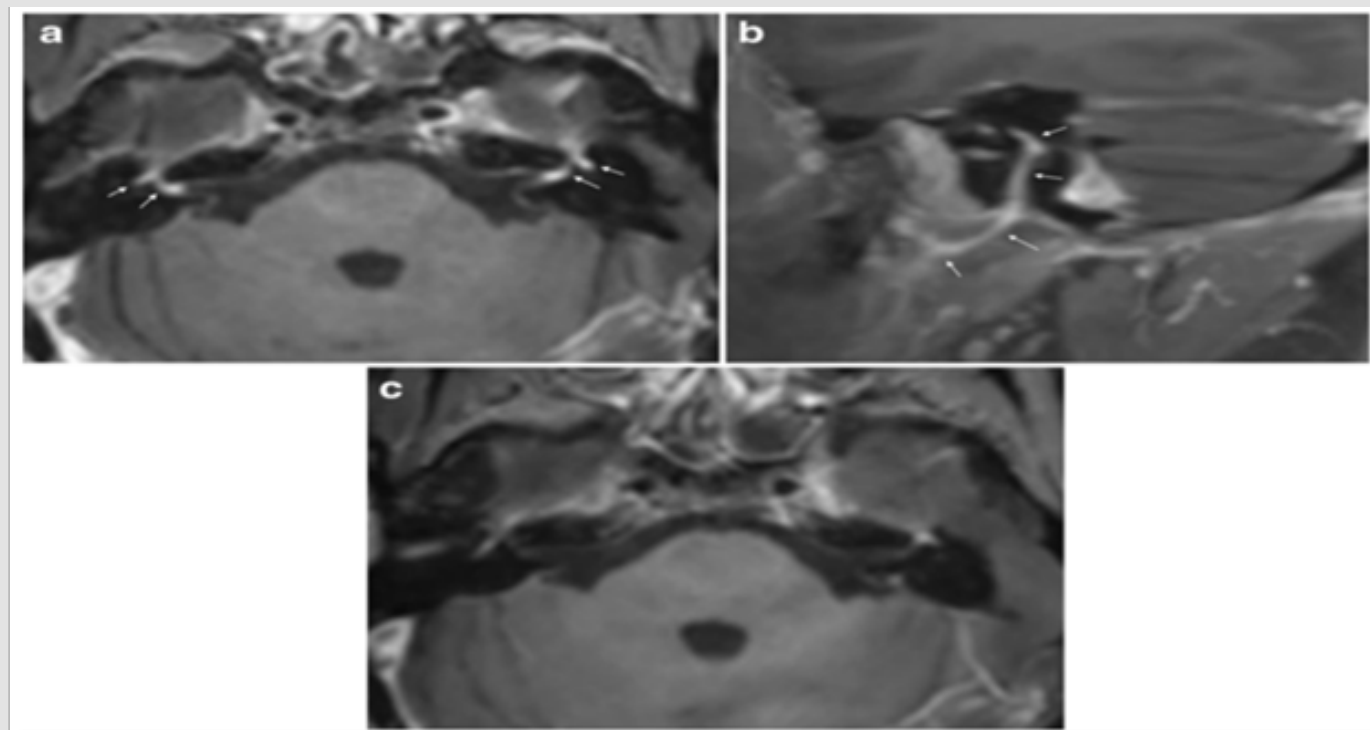

Figure 2: Axial post-contrast T1-weighted image

a) Showed intense enhancement in the distal intracanalicular, labyrinthine and geniculate ganglion segments of both facial nerves (arrows point to these structures mentioned from medial to lateral).

b) On the right side demonstrated the enhancement involving the tympanic and mastoid segment, as well as the parotid segment with the temporofacial and cervicofacial branches. Axial post-contrast fat suppressed T1-weighted image showed no abnormal enhancement of facial nerves 6 months after the treatment

c) T1 -weighted postcontrast MR image shows enhancement of the left facial nerve (arrow).

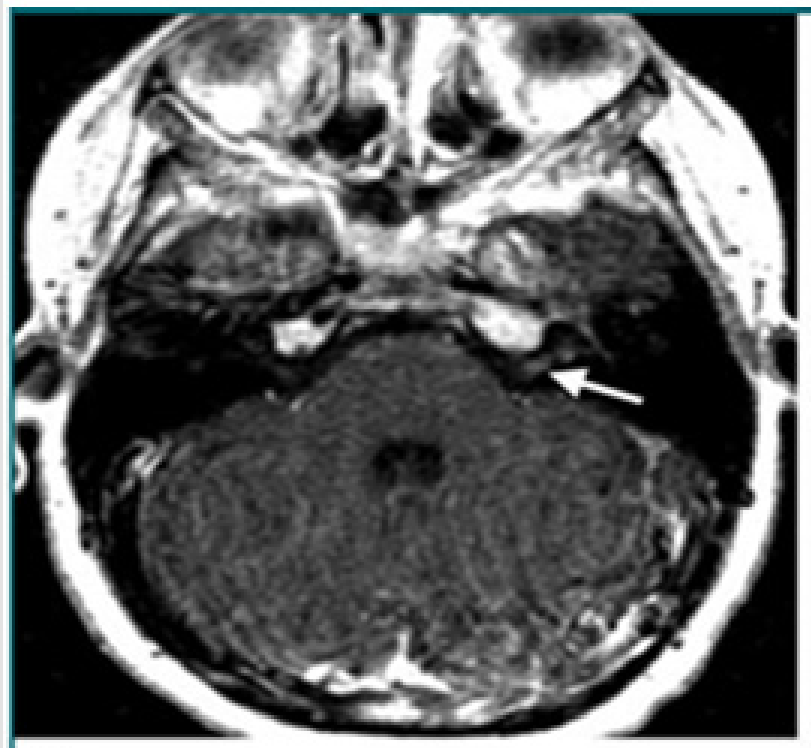

a.

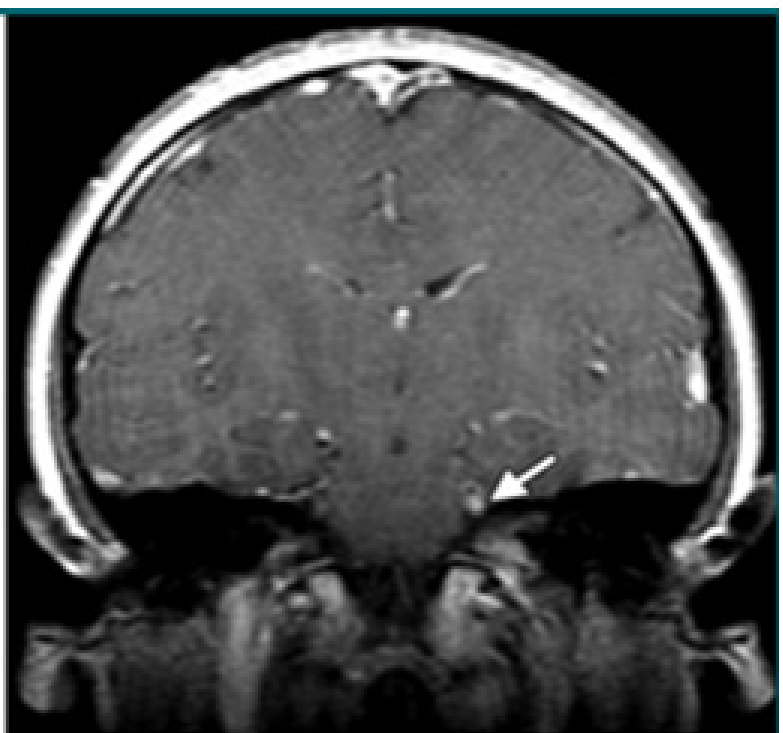

b.

Figure 3:

a) T1 -weighted postcontrast MR image shows enhancement of the left facial nerve (arrow),

b) Coronal post contrast $\mathrm{T} 1$ weighted MR image shows the left trigeminal nerve enhancement as well (arrow). 


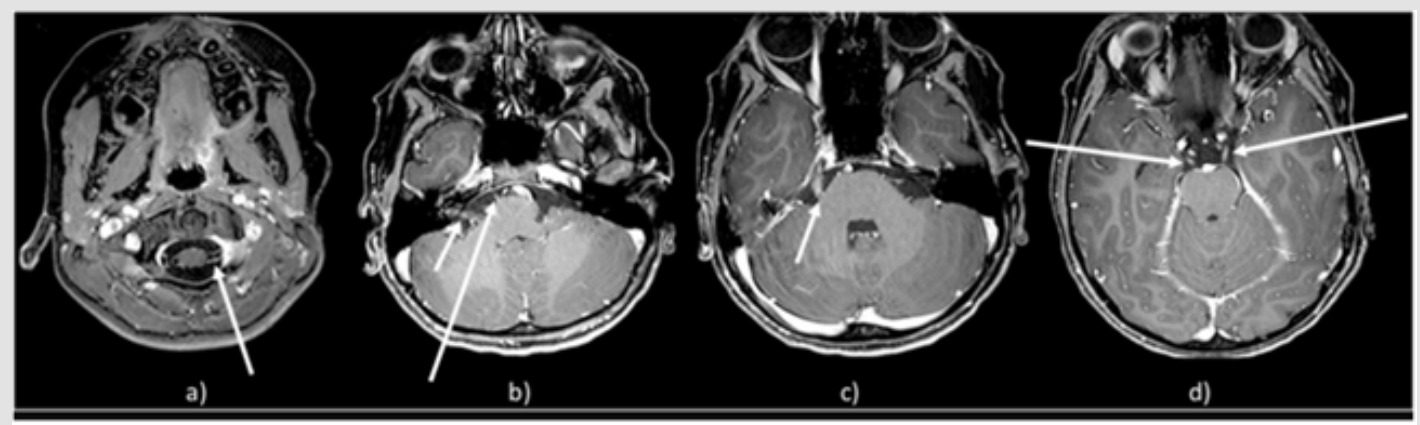

Figure 4: Post-gadolinium axial images demonstrate multiple enhancing nerves including

a) Ventral and dorsal cervical nerve roots

b) CN VII/VIII in acoustic canal and CN VI (cistern)

c) $\mathrm{CN} \mathrm{V} \mathrm{(cistern)}$

d) Bilateral CN VI.

\section{Lymphocytic Meningitis}

Between 19.5 - 29\% of LNB cases may develop meningitis as well $[1,19]$. Lymphocytic meningitis, with headache, meningeal signs, photophobia, and other symptoms, develops in less than $5 \%$ of Lyme disease. In acute and subacute cases with meningitis, the CSF routinely shows a pleocytosis (20 to 250 lymphocytes/mm3) with moderately elevated protein; the glucose concentration is usually normal but may be slightly depressed. [20].

\section{Meningoradiculitis}

The most common manifestation of nervous system involvement in European Lyme borreliosis is the Bannwarth syndrome that occurs in up $80 \%$ of patient [21]. Bannwarth syndrome accompanies with radiculoneuritis with severe radicular pain and paresis of extremities or the abdominal wall accompanied with CSF lymphocytic pleocytosis. The Meningoradiculitis is presented with a syndrome like sciatica with severe radicular pain toward the site of the tick bite. The Pain is usually severe, and is typically accompanied by corresponding sensory, motor, and reflex changes. The distribution involves one or a few adjacent roots. Bannwarth syndrome can involve the trunk; when it happens, similar to diabetic truncal neuropathy it can be misdiagnosed to a visceral disorder, leading to prolonged and wrong diagnostic approaches. That syndrome is rare in the USA[1,22].

Images in Figure 5show a case of LNB with meningoradiculitis where leptomeningeal enhancement was diffuse and widespread, and disappeared promptly with treatment.

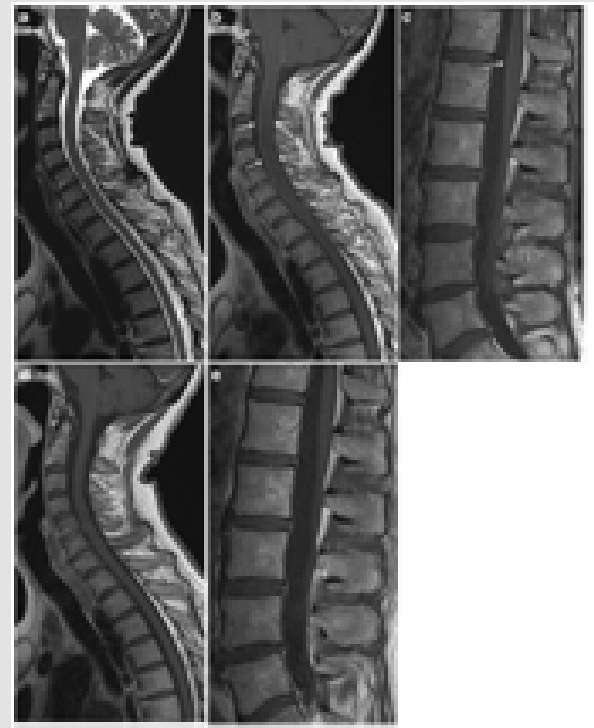

Figure 5: On the MRI, enhancement of cervical roots, thoracic and lumbar spinal nerve roots and leptomeningeal enhancement on post-contrast T1-weighted images, have been presented. $(23,14)$

Images in Fig. 6 show a case of LNB with meningoradiculitis where leptomeningeal enhancement was diffuse and widespread, and disappeared promptly with treatment. 
It is also important to be aware of the rather low specificity of meningeal enhancement with T1-weighted images, mainly due to interpretation disturbed by the normal enhancement in vascular structures. For bacterial meningitis in general, postcontrast T1weighted images yield a low rate of leptomeningeal enhancement, while the use of post-contrast FLAIR sequence increases sensitivity of MRI [20].Therefore, LNB should be considered in the list of causes of meningeal and nerve enhancement in endemic area and patients with a travel history of endemic areas, as they are common findings (Figure 6)[20]. There is a lack of correlation between enhancing cranial or radicular nerves and neurological symptoms as well.It is important to say that post-contrast FLAIR images have a higher specificity compared to contrast-enhanced T1 images in the detection of leptomeningeal enhancement.
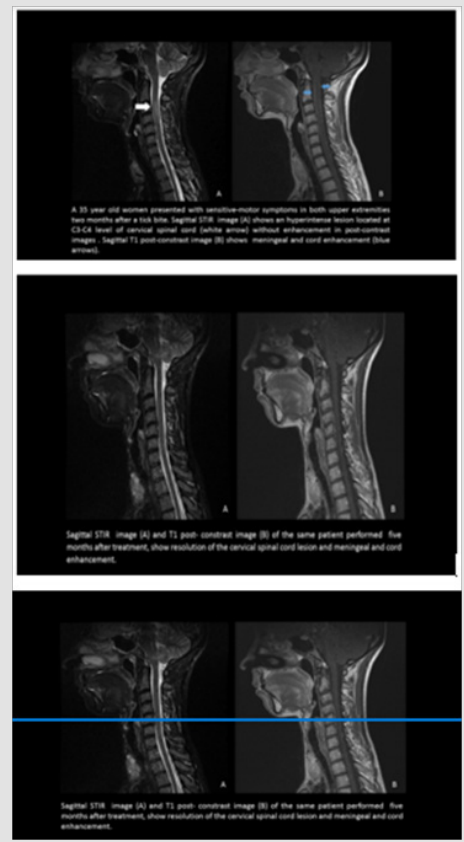

Figure 6: Complete resolution of myelopathy and spinal cord leptomeningeal enhancement after treatment.

Therefore, it can be a useful tool in the evaluation of infectious leptomeningitis as well[24,25]Additionally, the specificity of meningeal enhancement with T1-weighted images, mainly because of enhancement could be disturbed by the normal enhancement in vascular structures.

\section{Central Nervous System}

There are two different pathways that Borrelia species can reach the CNS. They reach to the CNS hematogeneously, or retrogradely via the peripheral nerves. The mechanism of central nervous system involvement possesses some explanations such as direct brain invasion, vasculitic processes or immunologic mechanisms [26]. Clinically, CNS symptoms can include headache, decrease the level of consciousness, and more severe encephalitis. In terms of MRI, approximately $50 \%$ of patients show multiple nonspecific T2 hyper-intense lesions in the subcortical and/or periventricular white matter similar to multiple sclerosis $[1,2,5]$ (Figure 7).Of course, the white matter lesions in middle-age and/ or elderly patients can mimic small vessel ischemia. MR findings were abnormal in $43 \%$ of the patients with LNB. Abnormal findings were exhibited in the cerebral white matter arcute fibers and brain stem as well[26,27,14]. The brain and cervical cord pathology in normal-appearing white matter, evaluated by magnetization transfer ratios and diffusion tensor imaging, are infrequent findings in patients with LNB [14]. Importantly, white matter involvement often persists on MR imaging despite successful treatment. Rarely, encephalomyelitis presented as tumefactive white matter ringenhancing lesions may simulate a neoplastic process [23].Another rare manifestation of LNB is vasculitis which may be associated with ischemic stroke, subarachnoid hemorrhage, and intracerebral hemorrhage [20].

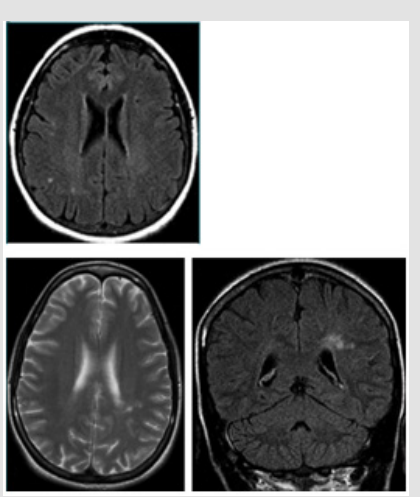

Figure 7: The Brain MRI findings of LNB. 


\section{Spinal Cord}

As mentioned before, the spinal cord involvement is rare. In spinal cord involvement, the MR imaging manifestations are multifocal or diffuse T2- weighted cord lesions and on postcontrast T1-weighted images nerve-root enhancement. In contrast to the classic cervical spinal cord MR imaging abnormalities seen in MS, most patients with LNB do not have macroscopic lesions or magnetization-transfer ratio changes[27-29]. Nerve root involvement is best seen on postcontrast T1-weighted sequences [30]. Spinal involvement has demonstrated diffuse or multifocal T2- weighted cord lesions and nerve root enhancement on postcontrast T1-weighted sequences (Figure 8a \& 8b) [40]. Therefore, the classical clinical LNB is manifestedby a predominant meningitis and radiculitis and the rare presence of intra-axial parenchymal brain and spinal cord involvement.It is important to indicate that although symptoms of LNB are usually nonspecific, however, in cases of root or meningeal enhancement, the symptoms and imaging findings were correlated. We sum up that meningeal and nerve-root enhancement is relatively common in LNB [14] (Figure 9).

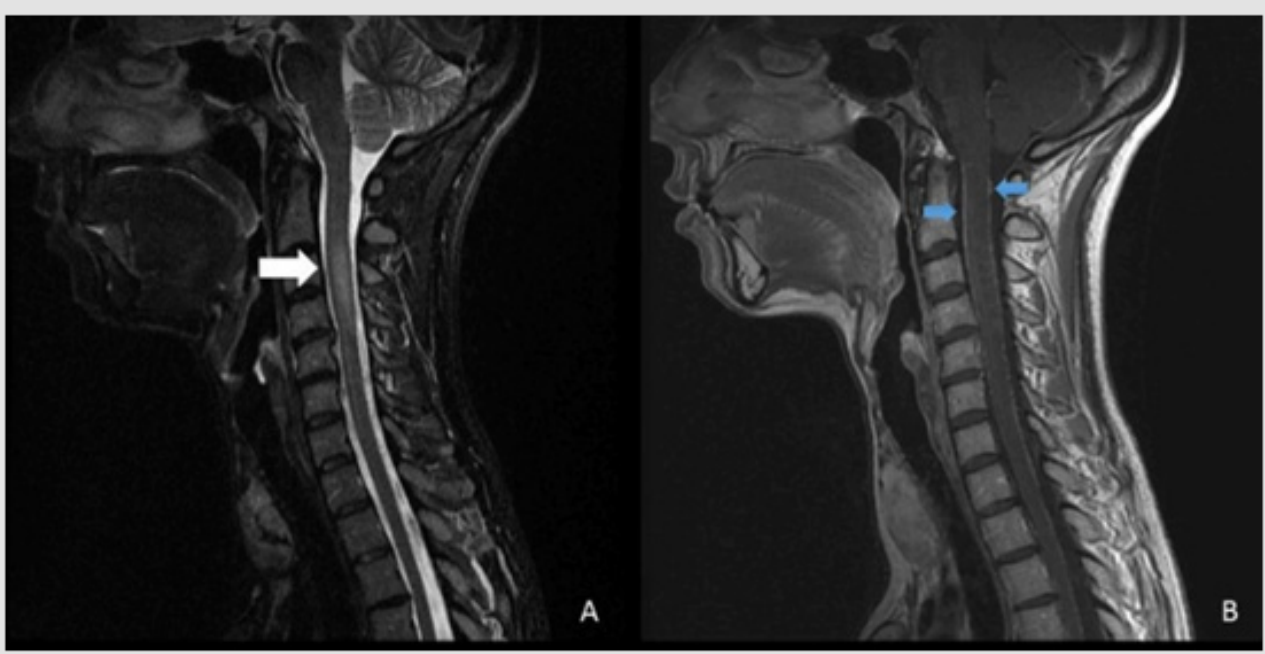

Figure 8a: A 35 year old woman with paresthesia and motor symptoms in upper limbs 2 months after a tick bit.

a) An hyperintense in C3-4 without enhancement

b) Meningeal enhancement.

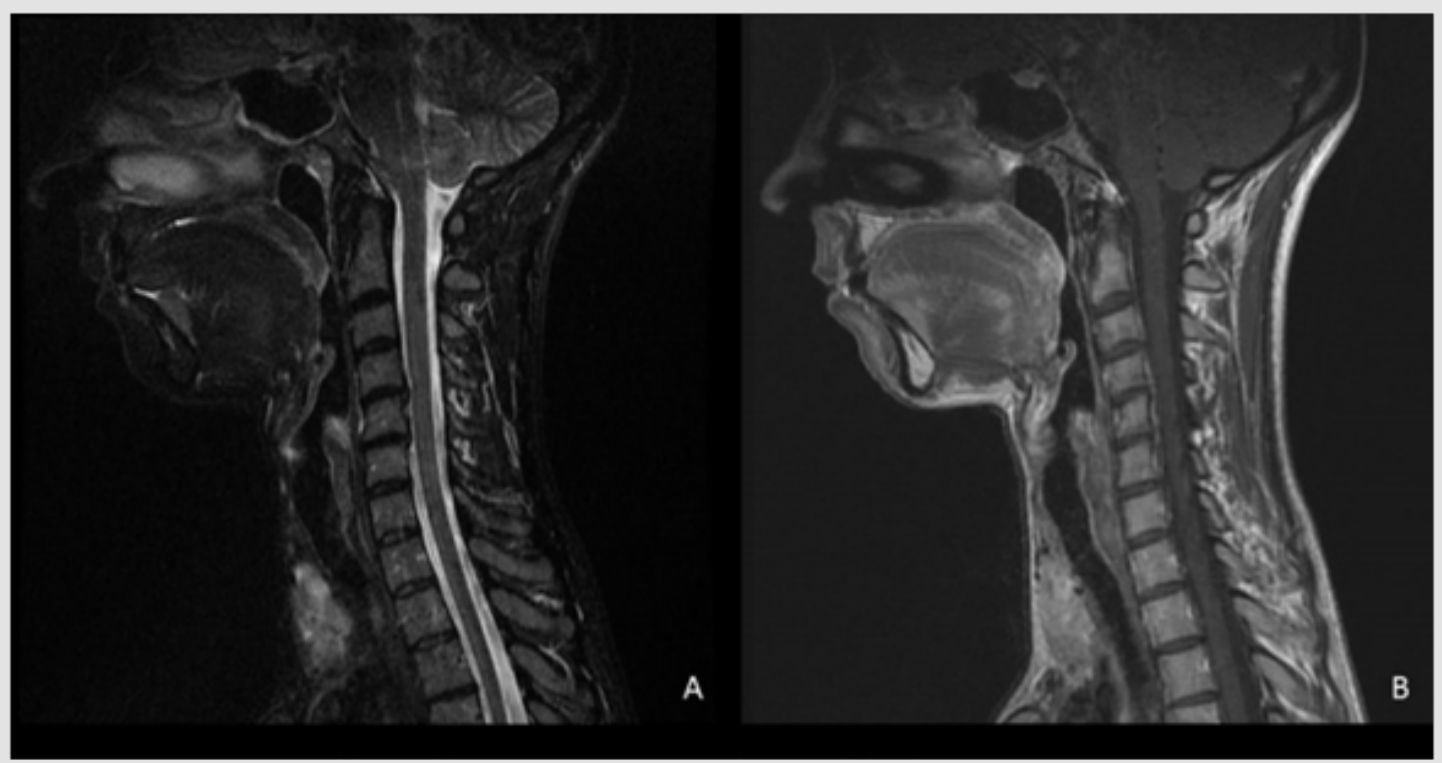

Figure 8b: (continue) The same patient after treatment. The cervical lesion and meningeal enhancement was disappeared [20]. 


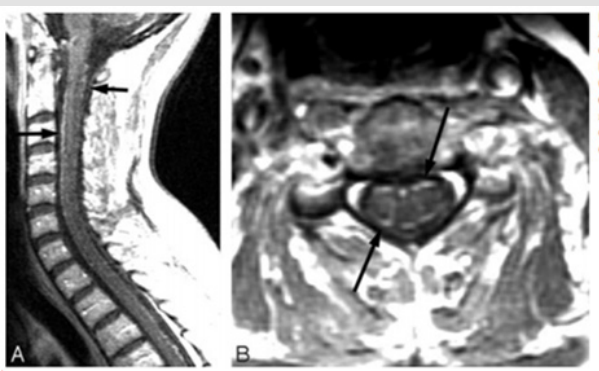

Figure 9: Meningeal involvement in Lyme.

\section{Ocular involvement in Lyme}

Ocular involvements are rare in LNB[31,32]. The different ocular involvement may happen in different stages of LNB. The most common ocular involvement in LNB is optic neuritis and uveitis. Conjunctivitis and episcleritis are commonly seen during the first stage[33,34]. But chronic intraocular inflammation, orbital myositis and keratitis are appeared in the third stage of Lyme disease. Lyme disease may closely be similar to pseudo-tumor cerebri as well (Figure 10) [14,35].
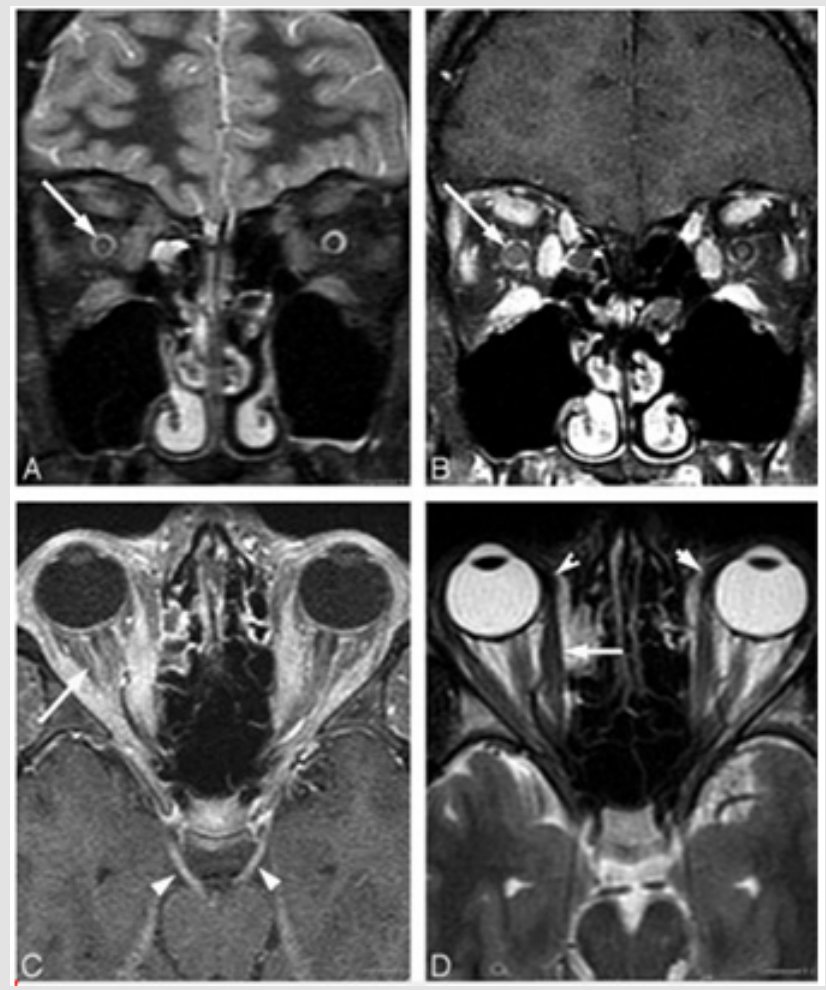

Figure 10: A 17-year-old - boy with papilledema and orbital pain and rule out of pseuotumor.

a) Right optic nerve edema on fat suppression T2 weighted fast spin- echo images.

b) Right greater than left optic nerve enhancement.

c) Bilateral third nerve cranial enhancement (arrowhead) and bilateral retrobulbar Component congestion.

d) Note the generalized extraocular muscular enlargement and enhancement including the insertions.

\section{Diagnosis}

In the first 4 weeks of infection, when the Erythema Migrants (EM) is present, biopsies of the relatively asymptomatic EM typically demonstrate innumerable spirochetes[1]. However, in this stage, in more than half of the patients the serum and CSF serologic assessments may be negative [36].The antibody response to B. burgdorferi infection develops slowly, and only 50 percent of patients in early-stage Lyme disease will have seropositive results. In other words, during the first month of infection, the humoral immune system has not prepared to combat with infection very well, since, the natural course of B cells immune system selection, multiplication and ultimately production of measurable quantities of specific antibodies takes 4-6 weeks, and therefore the immune tests could be seronegative. The immunoglobulin $\mathrm{M}$ (IgM) and immunoglobulin G (IgG) antibodies seemingly take two to four and four to six weeks and increase to peak level at six to eight weeks, respectively. Although, IgM usually drops to low levels after four to six months of infection, IgG rests present at low levels despite cure as well [37]. 
It is widely believed that in the first infection, the serologic tests overall, have very low sensitivity; however, by 4-6 weeks after infection, the sensitivity of tests is excellent.Academy of Neurology (AAN) diagnostic criteria include the presence of one or more of Erythema Migrans (EM), over microbiology or polymerase chain reaction (PCR). To upsurge specificity of serology, a twotier approach is suggested first a positive test of enzyme linked immunosorbent assays (ELISAs) followed by a test of Western blots if the primary goal is positive or borderline as well[38].Therefore, serum serology assessments should be made by ELISA methods within the four weeks of infection, if ELISA is positive, the samples should undergo Western blot testing for IgM and IgG; after four weeks, samples should be tested soley for IgG, as the risk of falsepositivewith IgM is high.

The specificity of this two-step approach is 59 and 95 percent in early and late stage of disease, respectively. When laboratory tests for Lyme disease is requested, physicians should be aware of reporting the duration of disease from the onset of infection.

In Western Blot test, 10 epitopes have been identified; the patients with IgG reactivity to any 5 are highly likely to have had Lyme borreliosis. This diagnostic approach is applied in the USA, but in Europe due to existence of multiple strains it could be more challenging. In addition to that, IgM Western blots are particularly problematic, because of two things: false positives are frequent and the broad cross reactivity of all IgM antibodies might occure. Significantly, since nearly all patients will develop IgG reactivity after about one month of infection, the diagnosis should be centered just on IgG levels and status[39]. The European Federation of Neurological Societies (EFNS) created guidelines for LNB diagnosis in 2010 [39]. The criteria for its diagnosis are: LNB compatible neurological symptoms and signs with no other explanation, pleocytosis and production of Borrelia burgdorferi specific antibodies (Bb) in the cerebrospinal fluid (CSF). Intrathecal antibody production is often presented as a positive $\mathrm{Bb}$ antibody index (AI), that evaluations of antibody level show a lower level in serum than CSF. According to the European guidelines, the infection is definite when all three criteria are met (Table 1). If two out of three criteria are present, the condition is possible too.

Table 1: EFNS diagnostic criteria for LNBa[39].

\begin{tabular}{|c|c|c|}
\hline Criteria & Definite LNB & Possible LNB \\
\hline $\begin{array}{l}\text { 1) Neurological symptoms } \\
\text { suggestive of LNB without } \\
\text { other obvious reasons }\end{array}$ & & \\
\hline $\begin{array}{l}\text { 2) Cerebrospinal fluid } \\
\text { pleocytosisb }\end{array}$ & & \\
\hline $\begin{array}{l}\text { 3) Intrathecal Bb antibody } \\
\text { production }\end{array}$ & & \\
\hline & All three criteria fulfilled & $\begin{array}{l}\text { Clinical criteria and one of } \\
\text { two laboratory criteria } \\
\text { fulfilled }\end{array}$ \\
\hline
\end{tabular}

Note:

EFNS European Federation of Neurological Societies, LNB Lyme neuroborreliosis

a Special criteria apply for late LNB with polyneuropathy [39]

$\mathrm{b}>5$ white blood cells per $\mathrm{mm}^{3}$

As mentioned previously, the sensitivity of tests is low in the early stage of infection, therefore, the explanation of serology results can be difficult in the infection diagnosis.It is important to say that both IgM and IgG antibodies may remain positive even several years after infection, so results may not show acute infection, previous exposure, or reinfection[39,40].Migration of micro-organisms into the CNS produces CXCL13 [41], a chemokine that attracts B. cells. Therefore, B. cells enter the CNS, and after multiplication, produce specific antibody against B. burgdorferi in the CSF [42]. It is important to note that the level of antibodies in the CSF should be corrected by measuring IgG in serum and CSF simultaneously, diluting both to the same final IgG concentration, then measuring the amount of specific antibody in each, looking at the ratio of the results. If the amount of antibody in CSF is more than expected from passive transfer from serum it means that the antibody level could be positive in the CSF[41,42]. The excess CSF antibody may carry on for a long time after treatment [43]. As for neurosyphilis, active infection is usually accompanied by elevated 
CSF protein or a CSF pleocytosis; both these data can be applied to think whether infection is currently active. Some studies further suggest that measurement of CSF CXCL13, though not necessarily specific for Lyme disease, may provide an additional useful marker of disease activity [43]. Recently, assessments of antibodies against the C6 peptide-a domain shared in different borrelia species including both European and US Lyme borreliosis-have shown to provide a very useful and promising new seroimmunology tool as well[43].

\section{Iranian approach for Lyme diagnosis}

In terms of Borreliosis, Iran is a low endemic area, therefore in Iran there are 3 scenarios:

a) The first one is a patient develops EM; the skin biopsy should be carried out to evaluate the presence of spirochetes.

b) The second scenario is if EM is absent but the patient has symptoms and signs of Lyme disease; the 2 tier serology approach has to be done. If the ELISA is negative, the Borreliosis is ruled out; whereas, if the ELISA is positive and Western blot is positive too, the diagnosis is confirmed.

c) In the last scenario, if EM and symptoms are not present but, in the screening, serology tests, the ELISA test is positive; we have to discard the tests and the test should be regarded as false positive!

False-negative results often are attributable to tests taken too early in the course of infection. Since, the antibody response develops slowly, tests taken within the first two weeks of infection have low sensitivities (less than 50 percent). Antibiotics also can influence the results of serologic tests when given early in the course of infection, and have been shown to abort seroconversion, even if inadequate therapy is provided. True seronegativity in the later stages of Lyme disease is uncommon [1].

\section{Treatment}

As a matter of fact, most patients with Lyme disease are likely to be treated if the disease is diagnosed immediately and given the appropriate treatments. If the disease is undiagnosed for months or years, a full improvement becomes less likely[19].

Lyme disease treatment depends on the different clinical status. We discuss the different status as below:

a) In uncomplicated Lyme disease: for adult, a two-week course of doxycycline, $100 \mathrm{mg}$ twice a day, but for Children over the age of eight can receive $4 \mathrm{mg} / \mathrm{kg} /$ day divided into twice daily dosing for two weeks[44].

b) In Lyme arthritis: doxycline $100 \mathrm{mg}$ twice a day for four weeks [45].

c) In Symptomatic Lyme carditis: hospitalization and intravenous ceftriaxone 2 gr divided dose daily in adults and until the symptoms have been resolved, then doxycycline should be started[44].Ceftriaxone, $2 \mathrm{~g} /$ day in adults and50$75 \mathrm{mg} / \mathrm{kg} /$ day in children.

d) In LNB: The decision depends upon whether or not meningitis is present or suspected. In meningitis approved by CSF analysis: intravenous ceftriaxone 2 gr divided dose daily in adults and $50-75 \mathrm{mg} / \mathrm{kg} /$ day in children. In suspected meningitis that cannot be confirmed by CSF, oral doxycycline up to 3 weeks $[44,45]$ (Table 2).

Table 2: The treatment options for LYME diseases.

\begin{tabular}{|c|c|}
\hline Lyme status & Treatment regimen \\
\hline Uncomplicated Lyme & Doxycyline $100 \mathrm{mg}$ oral bid for 2 weeks \\
\hline Lyme arthritis & Doxycycline $100 \mathrm{mg}$ oral bid for 4 weeks \\
\hline Lyme Carditis & Cefrixone IV $2 \mathrm{Gr}$ divided dose daily \\
\hline LNB & Cefrixone IV $2 \mathrm{Gr}$ divided dose daily \\
\hline Suspected meningitis & Docycycline $100 \mathrm{mg}$ oral bid for 3 weeks \\
\hline
\end{tabular}

It is important to note that cranial nerve deficits may persist for long time and can even be permanent.Treatment may take many months, or even years, but many adults are able to attain remission as well.The neurological diseases could be persisted for a long time if the infections are not treated properly and quickly. Unfortunately, no test exists to prove the infection is completely gone after treatment. Even patients who are in remission can sometimes have relapses, but these relapses are usually more easily treated than the initial infections[6].

\section{References}

1. Halperin JJ (2011) Neurologic manifestations of lyme disease. Current infectious disease reports. 13(4): 360-366.

2. Steere AC, Malawista SE, Hardin JA, Ruddy S, Askenase PW (1977) Erythema chronicum migrans and Lyme arthritis: the enlarging clinical spectrum. Annals of internal medicine 86(6): 685-698.

3. Benach JL, Bosler EM, Hanrahan JP, Coleman JL, Habicht GS, et al. (1983) Spirochetes isolated from the blood of two patients with Lyme disease. New England Journal of Medicine 308(13): 740-742.

4. Aberer E (2007) Lyme borreliosis-an update. JDDG: Journal der Deutschen Dermatologischen Gesellschaft 5(5): 406-414.

5. Control CfD (2007) Prevention. Lyme disease--United States, 20032005. MMWR Morbidity and mortality weekly report 56(23): 573.

6. Chaturvedi A, Baker K, Jeanmonod D, Jeanmonod R (2016) Lyme disease presenting with multiple cranial nerve deficits: report of a case. Case reports in emergency medicine 2016.

7. Chams Davatchi C (1997) The First Endemic Case of Lyme Borreliosis in Iran. Medical Journal of The Islamic Republic of Iran (MJIRI) 11(3): 237-239.

8. Adabi M, Rahmani FA, Ghasemi M (2004) Report of a case of Lyme disease in Mazandaran. 29: 21-25.

9. Siadati PTA (2006) A case of Lyme disease (Lyme Borreliosis). Acta Medica Iranica. 222-224.

10. Hengge UR, Tannapfel A, Tyring SK, Erbel R, Arendt G, Ruzicka T (2003) Lyme borreliosis. The Lancet infectious diseases 3(8): 489-500. 
11. Paul S, Song PI, Ogbechie OA, Sugai DY, Morley KW, et al. (2016) Vesiculobullous and hemorrhagic erythema migrans: uncommon variants of a common disease. International journal of dermatology. 55(2): e79-e82.

12. Pachner AR, Steiner I (2007) Lyme neuroborreliosis: infection, immunity, and inflammation. The Lancet Neurology 6(6): 544-552.

13. Steere AC (1995) Musculoskeletal manifestations of Lyme disease. The American journal of medicine. 98(4): 44S-51S.

14. Hildenbrand P, Craven D, Jones R, Nemeskal P (2009) Lyme neuroborreliosis: manifestations of a rapidly emerging zoonosis. American Journal of Neuroradiology. 30(6): 1079-1087.

15. Agarwal R, Sze G (2009) Neuro-lyme disease: MR imaging findings. Radiology. 253(1): 167-173.

16. Aiken AH (2010) Central nervous system infection. Neuroimaging Clinics. 20(4): 557-580.

17. Holzknecht MC, Salvado E, Pastor AS, Acosta LEG, MEZOSI O (2017) MR imaging findings in neuro-Lyme disease 2017. In: Holzknecht, Salvado E, Pastor AS, Acosta LEG, Mezosi 0 (Eds.), European Congress of Radiology 2017.

18. Akgoz A, Mukundan S, Lee TC (2012) Imaging of rickettsial, spirochetal, and parasitic infections. Neuroimaging Clinics 22(4): 633-657.

19. Chaturvedi A, Baker K, Jeanmonod D, Jeanmonod R (2016) Lyme disease presenting with multiple cranial nerve deficits: report of a case. Case reports in emergency medicine. 2016: 7218906

20. Allan H Ropper, Martin A ASamuels, Joshua P Klein, Sashank Prasad (2020) Lyme disease. Principles of Neurology. $11^{\text {th }}$ Edition: 1343-1344.

21. Ogrinc K, Lusa L, Lotrič Furlan S, Bogovič P, Stupica D, et al. (2016) Course and outcome of early European Lyme neuroborreliosis (Bannwarth syndrome): clinical and laboratory findings. Reviews of Infectious Diseases 63(3): 346-353.

22. Halperin JJ, Pass HL, Anand AK, Luft BJ, Volkman DJ (1988) Nervous system abnormalities in Lyme disease. Annals of the New York Academy of Sciences 539(1): 24-34.

23. Lindland ES, Solheim AM, Andreassen S, Quist Paulsen E, Eikeland R, et al. (2018) Imaging in Lyme neuroborreliosis. Insights into imaging 9(5): 833-844.

24. Lummel N, Koch M, Klein M, Pfister H, Brückmann H (2016) Spectrum and prevalence of pathological intracranial magnetic resonance imaging findings in acute bacterial meningitis. Clinical neuroradiology. 26(2): 159-167.

25. Parmar H, Sitoh YY, Anand P, Chua V, Hui F (2006) Contrast-enhanced flair imaging in the evaluation of infectious leptomeningeal diseases. European journal of radiology 58(1): 89-95.

26. Rupprecht TA, Koedel U, Fingerle V, Pfister HW (2008) The pathogenesis of lyme neuroborreliosis: from infection to inflammation. Molecular medicine 14(3): 205-212.

27. Rafto SE, Milton W, Galetta S, Grossman R (1990) Biopsy-confirmed CNS Lyme disease: MR appearance at $1.5 \mathrm{~T}$. American journal of neuroradiology 11(3): 482-484.

28. Fernandez RE, Rothberg M, Ferencz G, Wujack D (1990) Lyme disease of the CNS: MR imaging findings in 14 cases. American journal of neuroradiology. 11(3): 479-481.
29. Agosta F, Rocca M, Benedetti B, Capra R, Cordioli C (2006) MR imaging assessment of brain and cervical cord damage in patients with neuroborreliosis. American journal of neuroradiology 27(4): 892-894.

30. Hattingen E, Weidauer S, Kieslich M, Boda V, Zanella FE (2004) MR imaging in neuroborreliosis of the cervical spinal cord. European radiology 14(11): 2072-2075

31. Sibony P, Halperin J, Coyle P, Patel K (2005) Reactive Lyme serology in optic neuritis. Journal of neuro-ophthalmology 25(2): 71-82

32. Blanc F, Ballonzoli L, Marcel C, De Martino S, Jaulhac B (2010) Lyme optic neuritis. Journal of the neurological sciences 295(1-2): 117-119.

33. Stanek G, Fingerle V, Hunfeld KP, Jaulhac B, Kaiser R, et al. (2011) Lyme borreliosis: Clinical case definitions for diagnosis and management in Europe. 17(1): 69-79.

34. Bodaghi B (2007) Ocular manifestations of Lyme disease. Médecine et maladies infectieuses 37(7-8):518-22.

35. Lesser RL (1995) Ocular manifestations of Lyme disease. The American journal of medicine 98(4): 60S-2S.

36. Aguero Rosenfeld ME, Nowakowski J, Bittker S, Cooper D, Nadelman RB (1996) Evolution of the serologic response to Borrelia burgdorferi in treated patients with culture-confirmed erythema migrans. Journal of Clinical Microbiology 34(1): 1-9.

37. Johnson BJ (2006) Lyme disease: serologic assays for antibodies to Borrelia burgdorferi. Manual of Molecular and Clinical Laboratory Immunology, $7^{\text {th }}$ Edition: American Society of Microbiology pp. 493-500.

38. Trevejo R, Krause P, Sikand V, Schriefer M, Ryan R, et al. (1999) Evaluation of two-test serodiagnostic method for early Lyme disease in clinical practice. The Journal of infectious diseases 179(4): 931-938.

39. Mygland Å, Ljøstad U, Fingerle V, Rupprecht T, Schmutzhard E (2010) EFNS guidelines on the diagnosis and management of European Lyme neuroborreliosis. European journal of neurology. 17(1): 8-e4.

40. Halperin JJ, Logigian EL, Finkel MF, Pearl RA (1996) Practice parameters for the diagnosis of patients with nervous system Lyme borreliosis (Lyme disease). Neurology. 46(3): 619-627.

41. Marra CM, Tantalo LC, Sahi SK, Maxwell CL, Lukehart SA (2010) CXCL13 as a cerebrospinal fluid marker for neurosyphilis in HIV-infected patients with syphilis. Sexually transmitted diseases. 37(5): 283.

42. Sillanpää H, Skogman BH, Sarvas H, Seppälä IJ, Lahdenne P (2013) Cerebrospinal fluid chemokine CXCL13 in the diagnosis of neuroborreliosis in children. Scandinavian journal of infectious diseases 45(7): 526-530

43. Rupprecht T, Lechner C, Tumani H, Fingerle V (2014) CXCL13 als Biomarker der akuten Neuroborreliose. Der Nervenarzt 85(4): 459-464.

44. Wright WF, Riedel DJ, Talwani R, Gilliam BL (2012) Diagnosis and management of Lyme disease. American family physician. 85(11): 10861093.

45. Shapiro ED (2014) Lyme disease. New England Journal of Medicine. 370(18): 1724-1731. 
ISSN: $2574-1241$

DOI: 10.26717/BJSTR.2020.29.004796

SShahbeigi. Biomed J Sci \& Tech Res

(C) (P) This work is licensed under Creative

Submission Link: https://biomedres.us/submit-manuscript.php

\begin{tabular}{ll} 
BIOMEDICAL & Assets of Publishing with us \\
RESEARCHES & - Global archiving of articles \\
& - Immediate, unrestricted online access \\
\hline
\end{tabular}

